Arq. Bras. Med. Vet. Zootec., v.68, n.5, p.1112-1116, 2016

\title{
Cardiomiopatia arritmogênica do ventrículo direito em felino: relato de caso
}

\author{
[Arrhythmogenic right ventricular cardiomiopathy in a feline: case report] \\ P.S. Backschat, G.T. Goldfeder, F. Ampuero, A.M.D. Lacerda, M.H.M.A. Larsson \\ Faculdade de Medicina Veterinária e Zootecnia - Universidade de São Paulo - São Paulo, SP
}

\begin{abstract}
RESUMO
Felino de 3,3kg, fêmea, oito anos de idade, castrada, sem definição racial, foi atendida no Hospital Veterinário da Faculdade de Medicina Veterinária e Zootecnia da Universidade de São Paulo (HOVETUSP), com histórico de dispneia, prostração, hiporexia e consequente emagrecimento progressivo, havia uma semana. Foram realizados exames de sangue: hemograma com contagem de plaquetas, função renal, função hepática e dosagens séricas de sódio e potássio, eletrocardiograma (ECG), radiografia torácica, ecoDopplercardiograma, e os resultados dos exames indicaram diagnóstico presuntivo de cardiomiopatia arritmogênica do ventrículo direito. O diagnóstico foi confirmado pela necropsia e pela constatação da presença de tecido fibroadiposo no miocárdio no exame histopatológico, possibilitando, assim, o relato do primeiro caso de cardiomiopatia arritmogênica do ventrículo direito, em gato, no Brasil.
\end{abstract}

Palavras-chave: gato, dispneia, histopatológico

\begin{abstract}
A female $3.3 \mathrm{~kg}$ feline, eight years-old, spayed, without racial definition, was submitted to the Veterinary Hospital of the School of Veterinary Medicine and Animal Science, São Paulo University (HOVET-USP) with dyspnea, prostration, hyporexia, and consequent progressive weight loss a week ago. Blood count with platelet count, renal and hepatic profiles, and serum sodium and potassium, chest radiography, electrocardiogram (ECG), and echocardiography were performed and diagnosis of arrhythmogenic right ventricular cardiomyopathy was assumed. The diagnosis was confirmed by necropsy findings and by the presence of fibro-fatty tissue infiltration observed in histopathology, thus featuring the first report of arrhythmogenic right ventricular cardiomyopathy in a cat in Brazil.
\end{abstract}

Keywords: cat, dyspnea, histopathology

\section{INTRODUÇÃO}

A cardiomiopatia arritmogênica do ventrículo direito (CAVD) é uma doença miocárdica adquirida, caracterizada pela infiltração de tecido de reposição fibroadiposo no coração, descrita em humanos, cães e, mais raramente, em gatos (Fox et al., 2000; Protonotarios e Tsatsopoulou, 2004; Basso et al., 2009). Na medicina humana, é uma das doenças mais importantes quando o assunto é morte súbita, durante exercícios físicos de jovens atletas (Corrado et al., 2000; Gemayel et al., 2001; Basso et al., 2009), e o diagnóstico é baseado em critérios maiores e menores (McKenna et al., ,1984).. Num estudo realizado por Basso et al. (2004), 23 cães da raça Boxer, diagnosticados com CAVD, serviram de modelo para compreender os mecanismos patogênicos e clínicos complexos, responsáveis pela progressão da doença e morte súbita. Em 2013, Meurs et al. demonstraram que a mutação do gene estriatina pode ser uma das causas de CAVD.

Fox et al. (2000) descreveram, pela primeira vez, na espécie felina, estudo retrospectivo em que 12 felinos foram diagnosticados com a doença, clinicamente semelhante à de seres humanos e cães acometidos. $\mathrm{Na}$ Europa, mais especificamente no Reino Unido, os primeiros dois casos de CAVD, na espécie felina, foram relatados por Harvey et al. (2005), cujas monitorizações eletrocardiográficas 
ambulatoriais por 24 horas (sistema Holter) evidenciaram bloqueio atrioventricular completo e complexos ventriculares multiformes, e em um deles também se evidenciou taquicardia ventricular e fenômeno R sobre T. Em 2009, Ciaramella et al., na Itália, descreveram um caso de CAVD com envolvimento importante de ventrículo esquerdo num gato.

O objetivo do presente trabalho é, portanto, relatar o primeiro caso de cardiomiopatia arritmogênica do ventrículo direito na espécie felina em território nacional.

\section{CASUÍSTICA}

O presente relato de caso envolve um felino de $3,3 \mathrm{~kg}$, fêmea, oito anos de idade, castrada, sem definição racial, atendida no Hospital Veterinário da Faculdade de Medicina Veterinária e Zootecnia da Universidade de São Paulo (HOVET-USP), com histórico de dispneia, prostração, hiporexia e consequente emagrecimento progressivo, havia uma semana. $\mathrm{O}$ animal era domiciliado, porém com vários outros contactantes felinos.

Ao exame físico, observou-se animal apático, com subpeso, temperatura retal de $36,9^{\circ} \mathrm{C}$, mucosas normocoradas e desidratação moderada. Apresentava taquipneia devido à dispneia inspiratória de padrão restritivo, além de efusões pleural e abdominal, à ultrassonografia. A auscultação torácica revelou ruídos cardíacos e pulmonares hipofonéticos, devido à presença do líquido na cavidade pleural. Após drenagem, nenhuma alteração foi detectada, à auscultação, em campos pulmonares e área cardíaca. A frequência cardíaca era de $216 \mathrm{bpm}$ em correspondência à frequência de pulso. Pulso jugular positivo estava presente. À palpação abdominal, foi evidenciada hepatoesplenomegalia. Realizou-se a mensuração da pressão arterial sistólica pelo método Doppler, obtendo-se valor médio, de cinco aferições realizadas, de $60 \mathrm{mmHg}$, e, após cuidadosa prova de carga, a pressão arterial sistólica elevou-se ao valor de $90 \mathrm{mmHg}$.

Os líquidos cavitários foram analisados, ambos de aspecto serossanguinolento e compatíveis como sendo transudato modificado. Por meio de toracocentese, drenaram-se $175 \mathrm{~mL}$ de líquido pleural e, por paracentese abdominal, $300 \mathrm{~mL}$ de líquido peritoneal. $\mathrm{O}$ hemograma revelou presença de anemia normocítica normocrômica com discreta neutrofilia, sem desvio à esquerda, e a bioquímica sérica nenhuma alteração importante. A radiografia torácica evidenciou presença de efusão pleural, dificultando observação nítida da silhueta cardíaca, parênquima pulmonar sem alterações e homogeneidade em região epigástrica, compatível com efusão peritoneal. O exame ecoDopplercardiográfico revelou aumento importante de câmaras cardíacas direitas, sem envolvimento do lado esquerdo (Fig. 1), insuficiência valvar tricúspide de grau importante devido à dilatação ventricular direita e septo interatrial, com grau moderado, e septo interventricular, com grau discreto, de retificação em direção ao lado esquerdo; além disso, a pressão arterial pulmonar sistólica, estimada no ventrículo direito por meio da insuficiência tricúspide e utilizando a equação modificada de Bernoulli (Boon, 1998), foi de $45 \mathrm{mmHg}$, sendo assim, classificada como de grau discreto. Todas as valvas foram analisadas em relação ao aspecto e à movimentação de suas cúspides e nenhuma alteração foi constatada. O eletrocardiograma (ECG) demonstrou dados inconclusivos referentes à cardiomiopatia arritmogênica do ventrículo direito, sugerindo apenas sobrecarga de câmaras cardíacas direitas.

Pelas informações obtidas na anamnese, pelos dados observados ao exame físico e pelos resultados dos exames complementares, concluiu-se tratar-se de cardiomiopatia arritmogênica do ventrículo direito. Solicitou-se também o exame de monitorização ambulatorialsistema Holter-24 horas, e o tratamento instituído, de início, foi Pimobendan (0,3mg/kg/BID). O animal foi acompanhado durante três dias consecutivos para realização de drenagem de líquidos cavitários, no entanto, no terceiro dia, o proprietário optou pela eutanásia e o exame necroscópico foi realizado.

O exame post mortem confirmou que o coração era o único órgão afetado, em consonância com os achados ecocardiográficos. Macroscopicamente, o átrio e o ventrículo direito exibiam moderada dilatação das câmaras cardíacas, com evidente diminuição da espessura das paredes. Fragmentos do coração foram submetidos à técnica histológica, e as lâminas confeccionadas foram coradas em hematoxilina- 


\section{Backschat et al.}

eosina (HE) e tricrômico de Masson. O exame histopatológico do ventrículo direito revelou presença multifocal de discretas áreas contendo tecido colágeno denso e adipócitos maduros no miocárdio, substituindo os cardiomiócitos (Fig. 2a). Também há presença focal dessas áreas na parede septal de ventrículo esquerda (Fig. 2b). A coloração tricrômico de Masson foi utilizada para evidenciar e confirmar a presença do tecido fibroso em ambos os ventrículos (Fig. 3a e 3b).

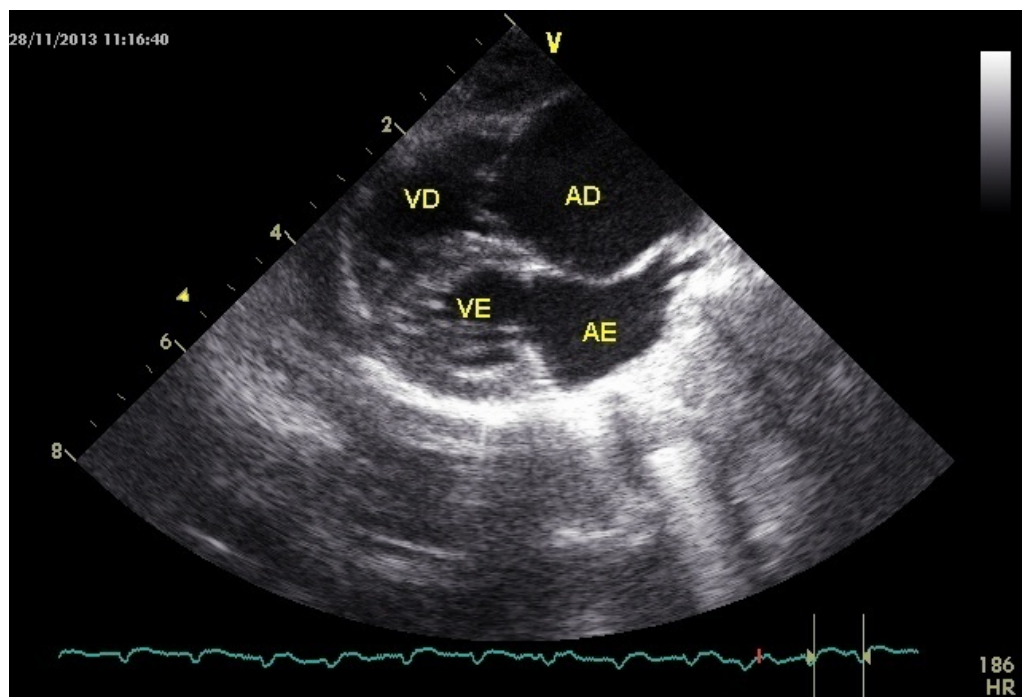

Figura 1. EcoDopplercardiograma de felino de 3,3kg, fêmea, oito anos de idade, castrada, sem definição racial, ilustrando aumento importante de câmaras cardíacas direitas sem envolvimento de câmaras esquerdas. Notar retificação de septo atrial de grau moderado em direção ao lado esquerdo. Corte longitudinal paraesternal direito (Fonte: Serviço de Cardiologia - HOVET - USP).
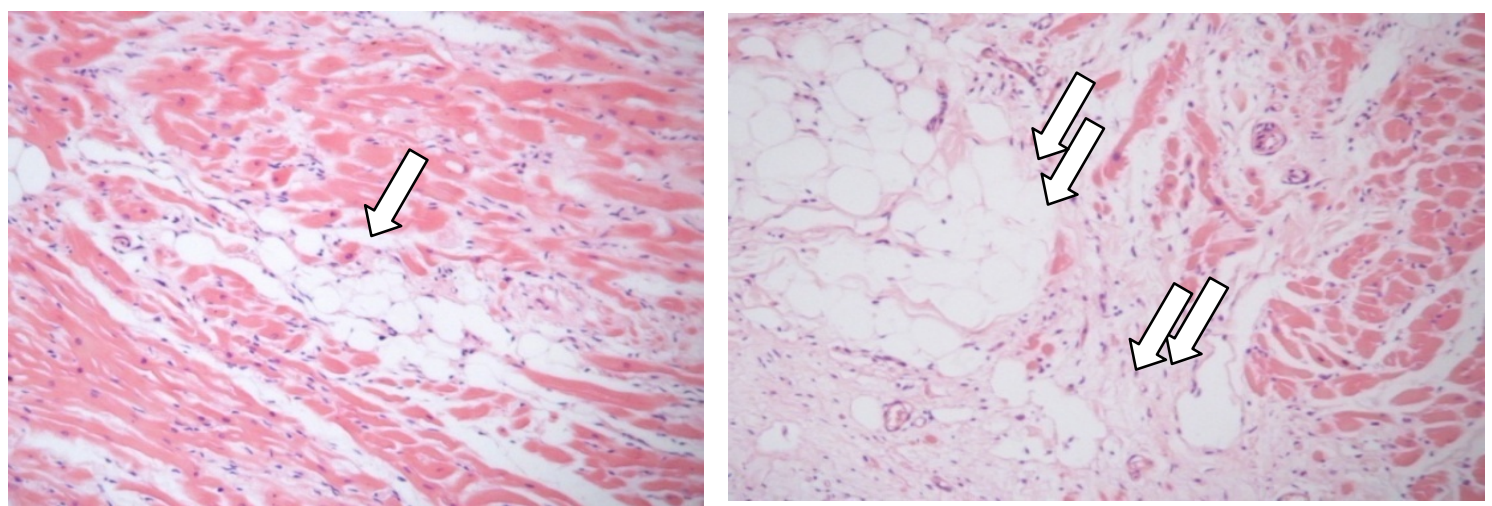

Figura $2 \mathrm{a}$ e $2 \mathrm{~b}$. Cortes histológicos de miocárdio de felino de $3,3 \mathrm{~kg}$, fêmea, oito anos de idade, castrada, sem definição racial. À esquerda, corte histológico de parede anterolateral de ventrículo direito, em região subtricuspídea, demonstrando o tecido colágeno denso e adipócitos entremeados ao miocárdio (setas). À direita, corte histológico de parede septal de ventrículo esquerdo, também demonstrando o tecido colágeno denso e adipócitos entremeados ao miocárdio. Coloração hematoxilina-eosina, aumento de 400x (Fonte: Serviço de Patologia Animal - HOVET- USP). 

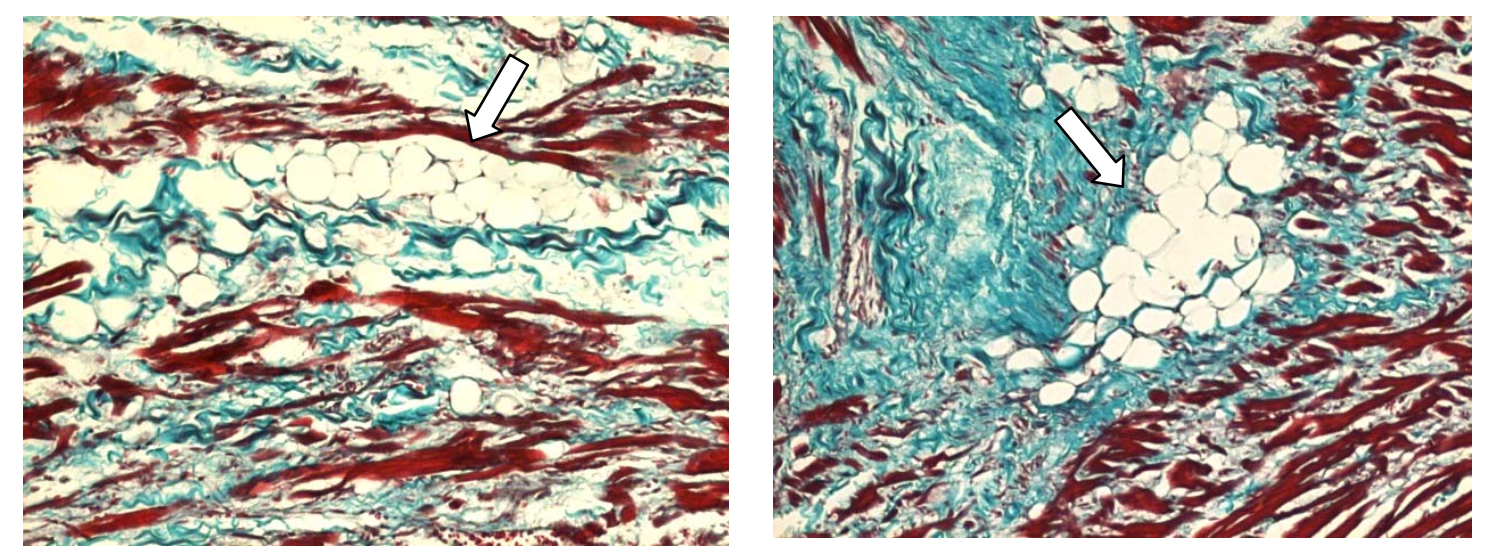

Figura 3a e 3b Cortes histológicos de miocárdio de felino de 3,3kg, fêmea, oito anos de idade, castrada, sem definição racial. À esquerda, corte histológico correspondente à parede anterolateral de ventrículo direito, em região subtricuspídea, e à direita parede septal de ventrículo esquerdo. As fibras colágenas coram-se em azul, enquanto as fibras musculares esqueléticas cardíacas coram-se em vermelho. Os adipócitos e a vacuolização da gordura intracitoplasmática não se coram pela técnica (setas). Coloração tricrômico de Masson, 10x (Fonte: Serviço de Patologia Animal - HOVET- USP).

Diante de todos esses achados, pode-se confirmar o primeiro caso de cardiomiopatia arritmogênica do ventrículo direito, com envolvimento, também, de ventrículo esquerdo, em gato, no Brasil.

O relato de caso apresentado está de acordo com as normas do Comitê de Ética no Uso de Animais da Faculdade de Medicina Veterinária e Zootecnia da Universidade de São Paulo, sob o seguinte número de aprovação: 1076120814.

\section{DISCUSSÃO}

O diagnóstico da CAVD em felinos, devido à baixa incidência, é de difícil padronização. As manifestações clínicas envolvidas são aquelas também encontradas na insuficiência cardíaca congestiva direita, como: pulso jugular positivo, hepatoesplenomegalia, dispneia devido às efusões cavitárias (pleural e/ou abdominal) e arritmias (Fox et al., 2000; Harvey et al., 2005; Ciaramella et al., 2009). Na medicina humana, o diagnóstico de CAVD é baseado em critérios maiores e menores, proposto por uma força-tarefa (McKenna et al., 1984). Entre os diagnósticos diferenciais, devem ser consideradas as anomalias congênitas relacionadas à câmara cardíaca direita, a exemplo da displasia de tricúspide e da anomalia de Uhl (Ciaramella et al., 2009).
O diagnóstico de CAVD do presente relato foi realizado com base no quadro sintomático, principalmente de insuficiência cardíaca congestiva direita, e exames complementares: radiografia torácica, eletrocardiografia, ecocardiografia e análise histopatológica (Fox et al., 2000; Harvey et al., 2005; Ciaramella et al., 2009).

Histologicamente, o tecido de reposição, caracterizado por tecido colágeno denso e adiposo, já avançava para o ventrículo esquerdo, corroborando com a declaração de Basso et al. (2009) de que o termo correto deveria ser "cardiomiopatia arritmogênica", uma vez que a mais nova proposta a respeito da patogênese da doença envolve erros a níveis genéticos, nos quais as proteínas desmossomais não promovem correta adesão celular, causando apoptose e, futuramente, tecido de reposição fibrogorduroso. Tal hipótese surgiu em torno de uma síndrome denominada síndrome de Naxos e de sua variante, síndrome de Carvajal (Protonotarios e Tsatsopoulou, 2004), em que os indivíduos acometidos desenvolvem uma queratodermia palmoplantar, cabelo lanoso e, posteriormente, a CAVD, em se tratando da síndrome de Naxos e da síndrome de Carvajal, que acomete o ventrículo esquerdo.

Trabalho recentemente realizado por Meurs et al. (2013) demonstrou que a mutação do gene estriatina, principalmente nos genótipos homozigotos, obteve uma forte associação nos 


\section{Backschat et al.}

29 Boxers positivos com a CAVD. No entanto, 3/33 dos Boxers com cardiomiopatia dilatada foram negativos para a mutação da estriatina, sugerindo que há, ainda, uma outra causa para o desenvolvimento da doença.

Ecocardiograficamente, os achados foram compatíveis com os achados macroscópicos da necropsia. Aumento de câmeras cardíacas direitas, insuficiência da valva tricúspide e espessura diminuída da parede de ventrículo direito são achados que corroboram os de outros autores (Fox et al., 2000; Harvey et al., 2005; Ciaramella et al., 2009).

Em relação ao exame necroscópico, o animal apresentava distensão abdominal volumosa (ascite), efusão pleural, e uma inspeção minuciosa de cavidade torácica foi realizada para exclusão de diagnóstico diferencial de neoplasias. Macroscopicamente, o lado direito do coração apresentava-se dilatado, e sua parede extremamente adelgaçada, denotando a marca da CAVD denominada "triângulo da displasia", caracterizada por regiões de aneurisma que ocorrem em três áreas mais especificamente, quais sejam: ápice, infundíbulo e subtricuspídea (Corrado et al., 2000; Gemayel et al., 2001; Basso et al., 2009). Quanto à histologia, os achados do tecido de reposição fibroadiposo evidenciaram o acometimento de ventrículo esquerdo, assemelhando-se com o caso relatado por Ciaramella et al. (2009).

\section{CONCLUSÃO}

A etiologia, bem como a patogenia da cardiomiopatia arritmogênica do ventrículo direito em felinos permanece obscura, principalmente devido à rara incidência da enfermidade na espécie. No Brasil, até então, a doença não havia sido diagnosticada na espécie felina; no caso em questão, foram necessários o exame post mortem e a análise histopatológica para observação do tecido de reposição fibroadiposo, confirmando, assim, o diagnóstico de cardiomiopatia arritmogênica do ventrículo direito, uma vez que o eletrocardiograma se mostrou inespecífico para as arritmias decorrentes dessa enfermidade. $\mathrm{O}$ animal do presente relato foi avaliado, eletrocardiograficamente, por ECG de rotina apenas, isto é, por alguns minutos; ressalta-se em caso de suspeita de CAVD a necessidade e a importância da realização da monitorização ambulatorial sistema Holter-24 horas para o diagnóstico presuntivo da enfermidade.

\section{REFERÊNCIAS}

BASSO, C.; CORRADO, D.; MARCUS, F.I. et al. Arrhythmogenic right ventricular cardiomyopathy. Lancet, v.373, p.1289-1300, 2009.

BASSO, C.; FOX, P.R.; MEURS, K. et al. Arrhythmogenic right ventricular cardiomyopathy causing sudden cardiac death in boxer dogs: a new animal model of human disease. Circulation, v.109, p.1180-1185, 2004.

BOON, J.A. Manual of veterinary echocardiography. Baltimore: Williams \& Wilkins, 1998. p.35-260.

CIARAMELLA, P.; BASSO, C.; DILORIA, A.; PIANTEDOSI, D. Arrhythmogenic right ventricular cardiomyopathy associated with severe left ventricular involvement in a cat. J. Vet. Cardiol., v.11, p.41-45, 2009.

CORRADO, D.; BASSO, C.; THIENE, G. Arrhythmogenic right ventricular cardiomyopathy: diagnosis, prognosis, and treatment. Heart, v.83, p.588-595, 2000.

FOX, P.R.; MARON, B.J.; BASSO, C. et al. Spontaneously occurring arrhythmogenic right ventricular cardiomyopathy in the domestic cat: a new animal model similar to the human disease. Circulation, v.102, p.1863-1870, 2000.

GEMAYEL, C.; PELLICCIA, A.; THOMPSON, P.D. Arrhythmogenic right ventricular cardiomyopathy. $J$. Am. Coll. Cardiol., v.38, p.1773-81, 2001.

HARVEY, A.M.; BATTERSBY, I.A.; FAENA, M. et al. Arrhythmogenic right ventricular cardiomyopathy in two cats. J. Small Anim. Pract., v.46, p.151-156, 2005 .

MCKENNA, W.J.; THIENE, G.; NAVA, A. et al. Diagnosis of arrhythmogenic right ventricular dysplasia/cardiomyopathy. Br. Heart J., v.71, p.215218,1994

MEURS, K.M.; STERN, J.A.; SISSON, D.D. et al. Association of dilated cardiomyopathy with the striatin mutation genotype in boxer dogs. J. Vet. Intern. Med., v.27, p.1437-1440, 2013.

PROTONOTARIOS, N.; TSATSOPOULOU, A. Naxos disease and Carvajal syndrome: cardiocutaneous disorders that highlight the pathogenesis and broaden the spectrum of arrhythmogenic right ventricular cardiomyopathy. Cardiovasc. Pathol., v.13, p.185-194, 2004. 